\title{
On the Properties of Metamodeling in OWL
}

\author{
Boris Motik \\ FZI Research Center for Information Technologies at the University of Karlsruhe, \\ Karlsruhe, Germany \\ motik@fzi.de
}

\begin{abstract}
A common practice in conceptual modeling is to separate the intensional from the extensional model. Although very intuitive, this approach is inadequate for many complex domains, where the borderline between the two models is not clear-cut. Therefore, OWL-Full, the most expressive of the Semantic Web ontology languages, allows combining the intensional and the extensional model by a feature we refer to as metamodeling. In this paper, we show that the semantics of metamodeling adopted in OWL-Full leads to undecidability of basic inference problems, due to free mixing of logical and metalogical symbols. Based on this result, we propose two alternative semantics for metamodeling: the contextual and the HiLog semantics. We show that $\mathcal{S H O I Q}$ - a description logic underlying OWL-DL - extended with metamodeling under either semantics is decidable. Finally, we show how the latter semantics can be used in practice to axiomatize the logical interaction between concepts and metaconcepts.
\end{abstract}

\section{Introduction}

A common practice in conceptual modeling is to separate the intensional from the extensional model of a domain. The intensional model is analogous to a database schema and it describes the general structure and the regularities of the world. The extensional model is analogous to a database instance and it describes a particular state of the world. Such a modeling style has also influenced the design of the Ontology Web Language (OWL) [14, the W3C standard for building ontologies in the Semantic Web. Namely, OWL provides concepts and properties for building the intensional model, and individuals and relationships among them for building the extensional model.

To better understand this duality, consider the following example, originally presented in [16; a similar example may be found in 15. A natural way to represent kinship between animal species is to organize them in a hierarchy of concepts. For example, the concept Bird represents the set of all birds, and the concept Eagle is a subconcept of Bird, stating that all eagles are birds. This is an example of intensional knowledge, as it is concerned with defining the general notions of birds and eagles. Knowledge about concrete animals is represented by extensional knowledge, e.g. by stating that the individual Harry is an instance of Eagle. Now the intensional knowledge implies that Harry is an Bird as well. 
However, one might also make statements about individual species, such as "eagles are listed in the IUCN Red List1 of endangered species." Notice an important distinction: we do not say that each individual eagle is listed in the Red List, but that the eagle species as a whole is. Hence, we introduce a concept RedListSpecies, and consider the relationship between RedListSpecies and Eagle. Making the former a superconcept of the latter is incorrect, as it would imply that Harry is a RedListSpecies - clearly an undesirable conclusion. It is better to say that Eagle is a type of RedListSpecies. Thus, RedListSpecies acts as a metaconcept for Eagle. The style of modeling which provides for metaconcepts is called metamodeling, and it can be used to build concise models if we precisely axiomatize the properties of metaconcepts. For example, by stating that "it is not allowed to hunt the individuals of species listed in the Red List", we formalize the logical properties of the metaconcept RedListSpecies, allowing us to deduce that "it is not allowed to hunt Harry."

The examples such as the one given above are often dismissed with an argument that "eagle as a species" and "eagle as a set of all individual eagles" are not the one and the same thing, and should not be referred to using the same symbol. Whereas an in-depth philosophical investigation might provide a more definitive answer, we simply observe that the word "eagle" in most people's minds invokes a notion of a "mighty bird of prey." The interpretation of this notion as a concept or as an individual is secondary and is often contextdependent, so using different symbols for the same intuitive notion makes the model unnecessarily complex.

Metamodeling is provided in OWL-Full, the most expressive language of the OWL family. However, its semantics is controversial, mainly because it is nonstandard, and therefore makes realizing practical reasoning systems difficult [5]. Therefore, OWL-DL was conceived as a "well-behaved" subset of OWL-Full by imposing the following restrictions: $(i)$ the sets of logical and metalogical symbols are strictly separated, $(i i)$ the sets of symbols used as concepts, roles and individuals are strictly separated, and ( $i i i)$ restrictions required to yield a decidable logic, such as the one on simple roles in number restrictions [7, are enforced. These restrictions make OWL-DL a syntactical variant of the $\mathcal{S H O I N}(\mathbf{D})$ description logic, which is known to be decidable. This is desirable since, to practically implement reasoners for expressive logics, advanced optimization techniques are essential, and these are much easier to develop if the logic is decidable [1-ch. 9].

Since it does not enforce ( $\mathrm{iii}$ ), OWL-Full is trivially undecidable. To obtain a decidable logic supporting metamodeling, it is natural to ask whether OWL-DL, extended with metamodeling in the style of OWL-Full, remains decidable. However, in Section 2 we show that even the basic description logic $\mathcal{A L C}$ becomes undecidable if restrictions $(i)$ and $(i i)$ are not enforced.

We analyze this undecidability result, and show that it is actually due to $(i)$, that is, to free mixing of logical and metalogical symbols. In a way, metamodeling in OWL-Full goes beyond its original purpose, and allows the user to tamper with

\footnotetext{
${ }^{1}$ http://www.redlist.org/
} 
the semantics of the modeling primitives themselves. Therefore, in Section 3 we present two alternative semantics for metamodeling: a contextual or $\pi$-semantics, which is essentially first-order, and a HiLog or $\nu$-semantics, which is based on HiLog [4] - a logic providing a second-order syntax for first-order logic. We show that, under some technical assumptions, both semantics can be combined with $\mathcal{S H O I} \mathcal{Q}$, a description logic underlying OWL-DL, yielding a decidable fragment of OWL-Full without increasing the complexity of reasoning. Furthermore, we present a resolution-based decision procedure for the $\mathcal{S H \mathcal { L }}$ fragment which, we believe, provides a basis for a practical implementation. Finally, in Section 4 we discuss the added expressivity of metamodeling on a concrete example. Technical details from this paper are presented in the technical report [9].

\section{Undecidability of Metamodeling in OWL-Full}

The semantics of OWL-Full [14] is quite technical, so we introduce $\mathcal{A} \mathcal{L C}$-Fullan extension the basic description logic $\mathcal{A L C}$ with metamodeling in the style of OWL-Full. We use rdf:, rdfs: and owl: for the standard namespace prefixes.

Definition 1. Let $V$ be the vocabulary set consisting of these symbols:

owl:Thing, owl:Nothing, rdf:type, rdfs:subClassOf, owl:sameAs, owl:differentFrom, owl:complement $O f$, owl:union $O f_{1}$, owl:unionOf $f_{2}$, owl:intersection $O f_{1}$, owl:intersection $O f_{2}$, owl:someValuesFrom, owl:allValuesFrom, owl:onProperty

Let $N$ be the set of names such that $V \subseteq N$. An $\mathcal{A L C}$-Full knowledge base $K B$ is a finite set of triples of the form $\langle s, p, o\rangle$, where $s, p, o \in N$.

An interpretation $I$ is a triple $\left(\triangle^{I},{ }^{I}, E X T^{I}\right)$, where $\triangle^{I}$ is a non-empty set, ${ }^{I}: N \rightarrow \triangle^{I}$ is a name interpretation function and $E X T^{I}: \triangle^{I} \rightarrow 2^{\triangle^{I} \times \triangle^{I}}$ is an extension function. Let $C E X T^{I}: \triangle^{I} \rightarrow 2^{\triangle^{I}}$ be the concept extension function defined as $\operatorname{CEXT}^{I}(x)=\left\{y \mid(y, x) \in \operatorname{EXT}^{I}\left(\right.\right.$ rdf:type $\left.\left.{ }^{I}\right)\right\}$. An interpretation $I$ is a model of $K B$ if it satisfies all conditions from Table 1. $K B$ is satisfiable if and only if a model of $K B$ exists.

$\mathcal{A L C}$-Full differs from OWL-Full in that: $(i)$ it does not provide concrete predicates, $(i i)$ it does not include the meta-level resources such as owl:Class, and $(i i i)$ it allows only binary union and intersection. These distinctions are not relevant for our undecidability proof. We use $\langle a \sqcup b, p, o\rangle$ as a syntactic shortcut for $\langle x, p, o\rangle,\left\langle x\right.$,owl:union $\left.O f_{1}, a\right\rangle$ and $\left\langle x\right.$,owl:union $\left.O f_{2}, b\right\rangle$, where $x$ is a fresh name. We use similar shortcuts for $\langle s, p, a \sqcup b\rangle$ and for $\sqcap$.

We show the undecidability of $\mathcal{A L C}$-Full by a reduction from the well-known domino tiling problem [3]. A domino system is a triple $\mathcal{D}=(D, H, V)$, where $D=\left\{D_{1}, \ldots, D_{n}\right\}$ is a finite set of domino types, and $H \subseteq D \times D$ and $V \subseteq D \times D$ are horizontal and vertical compatibility relations, respectively. A $\mathcal{D}$-tiling of an infinite grid is a function $t: \mathbb{N} \times \mathbb{N} \rightarrow D$ such that $t(0,0)=D_{0}$ and, for all $i, j \in \mathbb{N},(t(i, j), t(i, j+1)) \in H$ and $(t(i, j), t(i+1, j)) \in V$. For an arbitrary domino system $\mathcal{D}$, determining whether a $\mathcal{D}$-tiling exists is undecidable [3]. 
Table 1. Semantics of $\mathcal{A L C}$-Full

1. $\langle s, p, o\rangle \in K B$ implies $\left(s^{I}, o^{I}\right) \in \operatorname{EXT}^{I}\left(p^{I}\right)$

2. $\operatorname{CEXT}^{I}\left(\right.$ owl:Thing $\left.{ }^{I}\right)=\triangle^{I}$

3. $\operatorname{CEXT}^{I}\left(\right.$ owl:Nothing $\left.{ }^{I}\right)=\emptyset$

4. $(x, y) \in \operatorname{EXT}^{I}\left(r d f s: s u b C l a s s O f^{I}\right)$ implies $\operatorname{CEXT}^{I}(x) \subseteq \operatorname{CEXT}^{I}(y)$

5. $(x, y) \in \operatorname{EXT}^{I}$ (owl:sameAs $\left.{ }^{I}\right)$ implies $x=y$

6. $(x, y) \in \operatorname{EXT}^{I}$ (owl:differentFrom ${ }^{I}$ ) implies $x \neq y$

7. $(x, y) \in \operatorname{EXT}^{I}$ (owl:complementOf ${ }^{I}$ ) implies $\operatorname{CEXT}^{I}(x)=\triangle^{I} \backslash \operatorname{CEXT}^{I}(y)$

8. $(x, y) \in \operatorname{EXT}^{I}\left(\right.$ owl:union $\left.O f_{1}^{I}\right)$ and $(x, z) \in \operatorname{EXT}^{I}\left(\right.$ owl:union $\left.O f_{2}^{I}\right)$ imply $\operatorname{CEXT}^{I}(x)=\operatorname{CEXT}^{I}(y) \cup \operatorname{CEXT}^{I}(z)$

9. $(x, y) \in \operatorname{EXT}^{I}$ (owl:intersection $\left.O f_{1}^{I}\right)$ and $(x, z) \in \operatorname{EXT}^{I}$ (owl:intersectionOf $f_{2}^{I}$ ) imply $\operatorname{CEXT}^{I}(x)=\operatorname{CEXT}^{I}(y) \cap \operatorname{CEXT}^{I}(z)$

10. $(x, y) \in \operatorname{EXT}^{I}$ (owl:someValuesFrom $\left.{ }^{I}\right)$ and $(x, p) \in \operatorname{EXT}^{I}$ (owl:onProperty ${ }^{I}$ ) imply $\operatorname{CEXT}^{I}(x)=\left\{w \mid(w, z) \in \operatorname{EXT}^{I}(p) \wedge z \in \operatorname{CEXT}^{I}(y)\right\}$

11. $(x, y) \in \operatorname{EXT}^{I}$ (owl:allValuesFrom $\left.{ }^{I}\right)$ and $(x, p) \in \operatorname{EXT}^{I}$ (owl:onProperty ${ }^{I}$ ) imply $\operatorname{CEXT}^{I}(x)=\left\{w \mid(w, z) \in \operatorname{EXT}^{I}(p) \rightarrow z \in \operatorname{CEXT}^{I}(y)\right\}$

For a domino system $\mathcal{D}$, let $K B_{\mathcal{D}}$ be the $\mathcal{A} \mathcal{L C}$-Full knowledge base consisting of triples (1) - (9). Lemma 1 shows that satisfiability of $K B_{\mathcal{D}}$ exactly encodes the problem of deciding whether a $\mathcal{D}$-tiling exists.

$$
\begin{array}{r}
\left\langle D_{i} \sqcap D_{j}, \text { rdfs:subClassOf, owl:Nothing }\right\rangle \text { for } 1 \leq i<j \leq n \\
\left\langle\text { GRID, rdfs:subClassOf, } D_{1} \sqcup \ldots \sqcup D_{n}\right\rangle \\
\langle\text { NotGRID, owl:complementOf, GRID }\rangle \\
\left\langle D_{i}, \text { rdfs:subClassOf, } \alpha_{i}\right\rangle,\left\langle\alpha_{i}, \text { owl:onProperty, owl:allValuesFrom }\right\rangle \\
\left\langle\alpha_{i}, \text { owl:allValuesFrom, NotGRID } \sqcup \bigsqcup_{\left(D_{i}, d\right) \in H} d\right\rangle \text { for } 1 \leq i \leq n \\
\left\langle D_{i}, \text { rdfs:subClassOf, } \beta_{i}\right\rangle,\left\langle\beta_{i}, \text { owl:onProperty, rdf:type }\right\rangle \\
\left\langle\beta_{i}, \text { owl:allValuesFrom, NotGRID } \sqcup \bigsqcup_{\left(D_{i}, d\right) \in V} d\right\rangle \text { for } 1 \leq i \leq n \\
\langle G R I D, \text { owl:someValuesFrom, GRID }\rangle \\
\langle G R I D, \text { owl:onProperty, owl:allValuesFrom }\rangle \\
\langle G R I D, \text { owl:onProperty, rdf:type }\rangle \\
\langle G R I D, \text { rdfs:subClassOf, owl:allValuesFrom }\rangle \\
\langle\text { rdf:type, owl:sameAs, owl:onProperty }\rangle \\
\left\langle a_{0}, 0, r d f: \text { type, GRID } \sqcap D_{0}\right\rangle
\end{array}
$$

Lemma 1. $A \mathcal{D}$-tiling exists if and only if $K B_{\mathcal{D}}$ is satisfiable.

Proof. $(\Rightarrow)$ For a $\mathcal{D}$-tiling $t$, let $I$ be an interpretation depicted in Figure 1, with $\operatorname{CEXT}^{I}\left(G R I D^{I}\right)=\left\{a_{i, j}\right\}$ and $\operatorname{CEXT}^{I}\left(D_{k}^{I}\right)=\left\{a_{i, j} \mid t(i, j)=D_{k}\right\}$, for $i, j \geq 0$ and $1 \leq k \leq n$. The triples (3) - (5) encode the compatibility relations of $\mathcal{D}$ (including NotGRID into (3) and (4) ensures that compatibility is enforced only among instances of $G R I D)$. Hence, it is easy to see that $I$ is a model of $K B_{\mathcal{D}}$.

$(\Leftarrow)$ Let $I$ be a model of $K B_{\mathcal{D}}$. An excerpt of $I$ is shown in Figure 1 in which a triple $\langle s, p, o\rangle$ is represented as an arc pointing from the node $s$ to the 


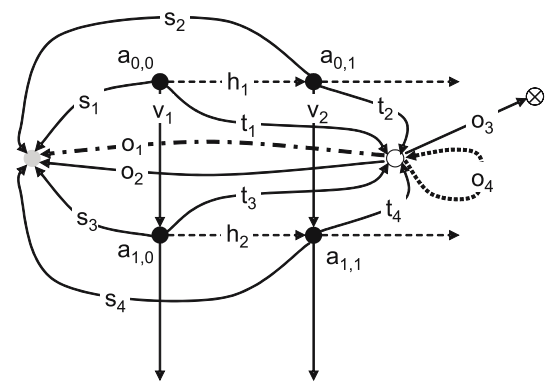

Legend:

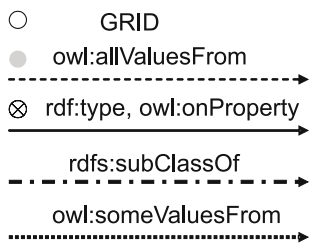

Fig. 1. Grid Structure in a Model of $K B_{\mathcal{D}}$

node $o$, whereas $p$ is encoded by the line type according to the legend. To refer easily to arcs, we assign them labels $t_{i}, h_{i}$ and $v_{i}$ (these do not correspond to $p$ ). For example, the arc $s_{1}$ represents the triple $\left\langle a_{0,0}\right.$, rdf:type, owl:allValuesFrom $\rangle$. Due to (10), rdf:type and owl:onProperty are synonyms, so $s_{1}$ also represents the triple $\left\langle a_{0,0}\right.$, owl:onProperty, owl:allValuesFrom $\rangle$. By an abuse of notation, we do not distinguish between the symbols and their interpretations.

Due to (11), $a_{0,0}$ is linked by $t_{1}$ to GRID. Due to (6), (77) and (8), $a_{0,0}$ is linked to $a_{0,1}$ and $a_{1,0}$ through $h_{1}$ and $v_{1}$, respectively, and $a_{0,1}$ and $a_{1,0}$ are in the concept extension of GRID by $t_{2}$ and $t_{3}$, respectively. Due to (6) and (7), $a_{1,0}$ is linked by $h_{2}$ to $a_{1,1}$, and by $t_{4}$ to GRID. Finally, by (9), all $a_{i, j}$ are in the concept extension of owl:allValuesFrom, that is, all $a_{i, j}$ have an $s_{l}$ arc to it.

Consider now the arcs at the node $a_{1,0}$. The arc $s_{3}$ can, due to (10), be read as $\left\langle a_{1,0}\right.$, owl:onProperty, owl:allValuesFrom $\rangle$. By applying Item 11 of Table 1 for $x=a_{1,0}$ and $y=a_{1,1}$, we conclude that, if $w$ is in the concept extension of $a_{1,0}$ and it is connected via $p=$ owl:allValuesFrom to some $z$, then $z$ must be in the concept extension of $a_{1,1}$. However, we may now set $w=a_{0,0}$ due to $v_{1}$, and $z=a_{0,1}$ due to $h_{1}$; this implies that $a_{0,1}$ is in the concept extension of $a_{1,1}$, that is, that $a_{0,1}$ is connected to $a_{1,1}$ by $v_{2}$. Hence, $a_{0,0}, a_{0,1}, a_{1,0}$ and $a_{1,1}$ are arranged in a two-dimensional grid, which continues indefinitely due to (6) - (8) .

A node $a_{i, j}$ in $I$ is allowed to have multiple owl:allValuesFrom and rdf:type successors, and all $a_{i, j}$ need not be distinct, so $I$ need not be a two-dimensional grid. However, a two-dimensional grid can easily be extracted from $I$ : one can choose any owl:allValuesFrom successor $a_{i, j+1}$ and any rdf:type successor $a_{i+1, j}$ of $a_{i, j}$, as well as any owl:allValuesFrom successor $a_{i+1, j+1}$ of $a_{i+1, j}$. Regardless of the choices, $a_{i, j+1}$ is always connected to $a_{i+1, j+1}$ by $r d f: t y p e$, so $a_{i, j}, a_{i, j+1}$, $a_{i+1, j}$ and $a_{i+1, j+1}$ are connected in a grid-like manner.

Hence, $I$ contains a two-dimensional infinite grid in which owl:allValuesFrom are horizontal, and rdf:type are vertical arcs. The triples (10) - (5) ensure that each grid node is assigned a single domino type corresponding to the compatibility relations $H$ and $V$ of $\mathcal{D}$, so a $\mathcal{D}$-tiling can easily be constructed from $I$.

Together with 3], Lemma 1 immediately implies the following result:

Theorem 1. Checking satisfiability of an $\mathcal{A L C}$-Full knowledge base $K B$ is undecidable. 


\section{Two Decidable Approaches to Metamodeling}

The proof of Lemma 1 reveals the causes for the undecidability of metamodeling in OWL-Full. Namely, this logic not only allows treating concepts as individuals, but it also allows mixing logical and metalogical symbols, and exposes its modeling primitives as individuals. We exploited this in axioms (5) and (6) of $K B_{\mathcal{D}}$, by stating an existential restriction on owl:allValuesFrom and rdf:type symbols and thus affecting their semantics. One would easily agree that tampering with the semantics of the ontology language is hardly desirable in practice, so in this section we present two alternative semantics for metamodeling.

In the following, we consider the description logic $\mathcal{S H O \mathcal { Q }}$, since it acts as the logical underpinning of the OWL family of languages. We do not consider datatypes here for the sake of simplicity. However, in [9] we show that, as long as datatypes are not subjected to metamodeling, they do not affect our results. We believe that this is not a practically relevant restriction: treating datatype individuals as concepts and vice versa will just unnecessarily confuse the users.

\subsection{The Syntax and Semantics of $\mathcal{S H O} \mathcal{I} \mathcal{Q}$ with Metamodeling}

Definition 2 (Syntax). For $N_{a}$ a set of atomic names, the set of names is defined as $N=N_{a} \cup\left\{n^{-} \mid n \in N\right\}$. For each $n \in N$, let $\operatorname{lnv}(n)=n^{-}$and $\operatorname{lnv}\left(n^{-}\right)=n$. A SHOI $R$ Box $K B_{\mathcal{R}}$ is a finite set of transitivity axioms $\operatorname{Trans}(R)$ and role inclusion axioms $R \sqsubseteq S$, where $R, S \in N$. As usual, we assume that $R \sqsubseteq S \in K B_{\mathcal{R}}$ implies $\operatorname{Inv}(R) \sqsubseteq \operatorname{Inv}(S) \in K B_{\mathcal{R}}$, and that $\operatorname{Trans}(R) \in K B_{\mathcal{R}}$ implies $\operatorname{Trans}(\operatorname{Inv}(R)) \in K B_{\mathcal{R}}$. Let $\sqsubseteq^{*}$ be the reflexive-transitive closure of $\sqsubseteq$. $A$ name $R$ is simple if for each name $S \sqsubseteq^{*} R$, Trans $(S) \notin K B_{\mathcal{R}}$. A set of $\mathcal{S H O I Q}$ concepts over $K B_{\mathcal{R}}$ is inductively defined as follows: each $A \in N$ is a concept and, for $R$ and $i$ names, $S$ a simple name, $C$ and $D \mathcal{S H O I Q}$ concepts and $n$ a non-negative integer, $\{i\}, \neg C, C \sqcap D, C \sqcup D, \exists R . C, \forall R . C, \geq n R . C$

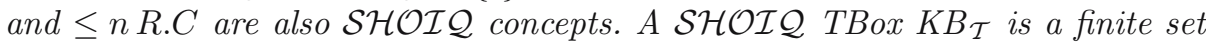
of concept inclusion axioms of the form $C \sqsubseteq D$, where $C$ and $D$ are $\mathcal{S H O I Q}$ concepts. $A \mathcal{S H O I \mathcal { Q } A B o x} K B_{\mathcal{A}}$ is a finite set of assertions of the form $C(a)$, $R(a, b)$ or (in) equality axioms of the form $a \circ b$, where $\circ \in\{\approx, \not{\neq}\}, C$ is a $\mathcal{S H O I} \mathcal{Q}$ concept, and $R$, a and $b$ are names. $A \mathcal{S H O I Q ~ k n o w l e d g e ~ b a s e ~} K B$ is a triple $\left(K B_{\mathcal{R}}, K B_{\mathcal{T}}, K B_{\mathcal{A}}\right)$. The logic $\mathcal{A L C H O \mathcal { Q }}$ is a fragment of $\mathcal{S H O I Q}$ without

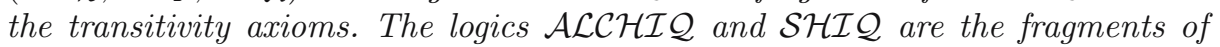
$\mathcal{A L C H O I Q}$ and $\mathcal{S H O I Q}$, respectively, without the nominal concepts $\{i\}$.

The major difference of Definition 2 to the usual definitions is that the sets of concept, role and individual names are not disjoint, but are merged into one set of names. We denote with $N_{K B}$ the subset of those names that occur in $K B$, and with $|K B|$ the size of $K B$ with the numbers coded in unary. We now define the so-called contextual semantics for $\mathcal{S H O I Q}$. 


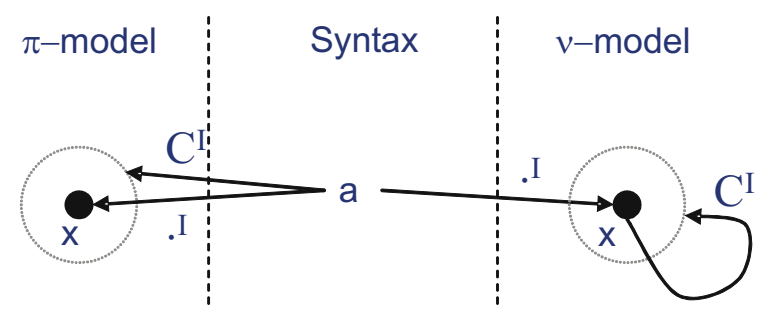

Fig. 2. $\pi$ - and $\nu$-models of the Axiom $a(a)$

Definition 3 (Contextual Semantics). For a $\mathcal{S H O I Q ~ k n o w l e d g e ~ b a s e ~ K B , ~ a ~}$ $\pi$-interpretation $I$ is a 4-tuple $\left(\triangle^{I},{ }^{I}, C^{I}, R^{I}\right)$ where $\triangle^{I}$ is a non-empty domain set, ${ }^{I}: N \rightarrow \triangle^{I}$ is a name interpretation function, $C^{I}: N \rightarrow 2^{\triangle^{I}}$ is an atomic concept extension function and $R^{I}: N \rightarrow 2^{\triangle^{I} \times \triangle^{I}}$ is a role extension function. The function $C^{I}$ is extended to concepts as specified in Table 2 , upper left section, where symbols are interpreted contextually, that is, depending on their syntactic position. A $\pi$-interpretation $I$ is a $\pi$-model of $K B$ if it satisfies all conditions from Table囵, lower left section. The notions of $\pi$-satisfiability, $\pi$-unsatisfiability and $\pi$-entailment (written $\models_{\pi}$ ) are defined as usual.

The contextual semantics is essentially equivalent to the one from 4 and to standard first-order semantics. Namely, in a first-order formula, the role of a symbol can be inferred from the place at which the symbol occurs in a formula, so the set of constant, function and predicate symbols need not be disjoint. We use $\pi$-semantics mainly as a baseline for a comparison with the HiLog semantics, defined below. This semantics is more in the spirit of OWL-Full, and is based on HiLog [4].

Definition 4 (HiLog Semantics). For a SHOIQ knowledge base KB, a $\nu$ interpretation $I$ is a 4-tuple $\left(\triangle^{I},{ }^{I}, C^{I}, R^{I}\right)$ where $\triangle^{I}$ is a non-empty domain set, ${ }^{I}: N \rightarrow \triangle^{I}$ is a name interpretation function, $C^{I}: \triangle^{I} \rightarrow 2^{\triangle^{I}}$ is an atomic concept extension function, and $R^{I}: \triangle^{I} \rightarrow 2^{\Delta^{I} \times \triangle^{I}}$ is a role extension function. The extension of the function $C^{I}$ to concepts and the interpretation of axioms are specified in Table Q right section. The notions of $\nu$-satisfiability, $\nu$-unsatisfiability and $\nu$-entailment (written $\models_{\nu}$ ) are defined as usual.

To understand the essential difference between these two semantics, consider the knowledge base $K B$ containing only the axiom $a(a)$, where the symbol $a$ is used both as an individual and as a concept. A $\pi$-model of $K B$ is depicted on the left-hand side of Figure 2 both the individual interpretation ${ }^{I}$ and the concept interpretation $C^{I}$ are assigned directly to the symbol $a$. A $\nu$-model of $K B$ is depicted on the right-hand side of Figure 2, the individual interpretation ${ }^{I}$ assigns the domain individual $x$ to the symbol $a$; however, the concept interpretation is not assigned to $a$, but to $x$. We discuss the consequences that such a definition of semantics has on entailment in Section 4 . 


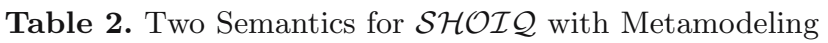

\begin{tabular}{|c|c|c|}
\hline & $\pi$-semantics & $\nu$-semantics \\
\hline \multicolumn{2}{|r|}{ Extending $C^{I}$ to concepts } & \multirow{14}{*}{$\begin{array}{l}C^{I} \text { and the interpretation of } \\
\text { axioms are obtained from the } \\
\text { ones for } \pi \text {-semantics by apply- } \\
\text { ing the following changes: } \\
\qquad C^{I}(A) \rightsquigarrow C^{I}\left(A^{I}\right)\end{array}$} \\
\hline $\begin{array}{c}A \\
\{i\} \\
\neg D\end{array}$ & $\begin{array}{c}C^{I}(A) \subseteq \triangle^{I} \\
\left\{i^{I}\right\} \\
\triangle^{I} \backslash C^{I}(D)\end{array}$ & \\
\hline$D_{1} \sqcap D_{2}$ & $C^{I}\left(D_{1}\right) \cap C^{I}\left(D_{2}\right)$ & \\
\hline $\begin{array}{l}D_{1} \sqcup D_{2} \\
\quad \exists S \cdot D \\
\quad \forall S D\end{array}$ & $\begin{array}{c}C^{I}\left(D_{1}\right) \cup C^{I}\left(D_{2}\right) \\
\left\{x \mid(x, y) \in R^{I}(S) \wedge y \in C^{I}(D)\right.\end{array}$ & \\
\hline $\begin{aligned} & \forall S . D \\
\leq & n S . D\end{aligned}$ & $\begin{array}{c}\left\{x \mid(x, y) \in R^{I}(S) \rightarrow y \in C^{I}(D)\right. \\
\left\{x \mid \sharp\left\{y \mid(x, y) \in R^{I}(S) \wedge y \in C^{I}(D)\right\} \leq n\right\}\end{array}$ & \\
\hline$\geq n S . D$ & $\left\{x \mid \sharp\left\{y \mid(x, y) \in R^{I}(S) \wedge y \in C^{I}(D)\right\} \geq n\right\}$ & \\
\hline \multicolumn{2}{|r|}{ Interpretation of axioms } & \\
\hline & $R^{I}(S)=R^{I}(\operatorname{Inv}(S))^{-}$ & \\
\hline$S \sqsubseteq T$ & $R^{I}(S) \subseteq R^{I}(T)$ & \\
\hline $\begin{array}{l}D_{1} \sqsubseteq D_{2} \\
\operatorname{Trans}(S)\end{array}$ & $\begin{array}{c}C^{I}\left(D_{1}\right) \subseteq C^{I}\left(D_{2}\right) \\
R^{I}(S)^{+} \subset R^{I}(S)\end{array}$ & \\
\hline$D(a)$ & $a^{I} \in C^{I}(D)$ & \\
\hline$S(a, b)$ & $\left(a^{I}, b^{I}\right) \in R^{I}(S)$ & \\
\hline$a \approx b$ & $a^{I}=b^{I}$ & \\
\hline$a \not \approx b$ & $a^{I} \neq b^{I}$ & \\
\hline
\end{tabular}

Note: $\sharp S$ is the number of elements in $S, S^{+}$is the transitive closure of $S$, and $S^{-}$is the inverse relation of $S$.

Neither semantics requires different names to be interpreted as different domain objects. If this is required, the unique name assumption should be axiomatized explicitly, by introducing an axiom $n_{i} \not \approx n_{j}$ for each $n_{i}, n_{j} \in N, n_{i} \neq n_{j}$.

Since the contextual semantics is essentially first-order, it can be decided using known algorithms, such as [8]. Therefore, we focus on deciding $\nu$-satisfiability.

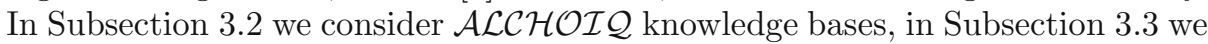
discuss the problems introduced by transitivity axioms, and in Subsection 3.4 we present a resolution-based practical decision procedure for the $\mathcal{A L C H \mathcal { C }}$ fragment.

\subsection{Deciding $\nu$-Satisfiability of $\mathcal{A L C H O I \mathcal { O }}$}

An equivalence relation $\mathcal{E}$ over a set of names $N$ induces a set or equivalence classes, so for each equivalence class we may arbitrarily select one representative name from it. For a name $n$, let $n / \mathcal{E}$ denote the representative name chosen for

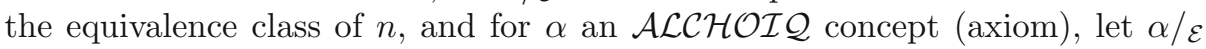
denote the concept (axiom) obtained from $\alpha$ by replacing each name $n$ with $n / \mathcal{E}$. Finally, let $K B$ be an $\mathcal{A L C H O \mathcal { O }}$ knowledge base and $\mathcal{E}$ an equivalence relation over $N_{K B}$; then $K B / \mathcal{E}$ is the knowledge base obtained from $K B$ by $(i)$ replacing each axiom $\alpha$ with $\alpha / \mathcal{E}$, and by (ii) appending an axiom $n_{i} / \mathcal{E} \not \approx n_{j} / \mathcal{E}$ for each pair of names $n_{i}, n_{j} \in N_{K B}$ such that $n_{i} / \mathcal{E} \neq n_{j} / \mathcal{E}$.

An algorithm for checking $\nu$-satisfiability of $K B$ can be easily obtained by non-deterministically guessing an equivalence relation $\mathcal{E}$ over $N_{K B}$, and then by 
checking $\pi$-satisfiability of $K B / \mathcal{E}$. The correctness of the algorithm is demonstrated by the following lemma:

Lemma 2. An $\mathcal{A L C H O I Q ~ k n o w l e d g e ~ b a s e ~} K B$ is $\nu$-satisfiable if and only if an equivalence relation $\mathcal{E}$ over $N_{K B}$ exists, such that $K B / \mathcal{E}$ is $\pi$-satisfiable.

Proof. $(\Leftarrow)$ Let $\mathcal{E}$ be an equivalence relation over $N_{K B}$ and $I^{\pi}$ a $\pi$-model of $K B / \mathcal{E}$. We construct a $\nu$-interpretation $I_{\nu}$ by setting $\triangle^{I_{\nu}}=\triangle^{I_{\pi}}, n^{I_{\nu}}=(n / \mathcal{E})^{I_{\pi}}$, $C^{I_{\nu}}\left(n^{I_{\nu}}\right)=C^{I_{\pi}}(n / \mathcal{E}), R^{I_{\nu}}\left(n^{I_{\nu}}\right)=R^{I_{\pi}}(n / \mathcal{E})$, for each $n \in N_{K B}$ and, finally, $C^{I_{\nu}}(x)=C^{I_{\pi}}(x)$ and $R^{I_{\nu}}(x)=R^{I_{\pi}}(x)$ for each $x \in \triangle^{I_{\nu}}$ such that there is no $n \in N_{K B}$ with $n^{I_{\nu}}=x$. Due to inequality axioms $n_{i} / \mathcal{E} \not n_{j} / \mathcal{E}$, we have $I_{\pi}\left(n_{i} / \mathcal{E}\right) \neq I_{\pi}\left(n_{j} / \mathcal{E}\right)$, so the construction assigns a unique value to $C^{I_{\nu}}(x)$ and $R^{I_{\nu}}(x)$ for each $x \in \triangle^{I_{\nu}}$, and $I_{\nu}$ is correctly defined. Furthermore, for each concept $X, C^{I_{\nu}}(X)=C^{I_{\pi}}(X / \mathcal{E})$, so $I_{\nu}$ is obviously a $\nu$-model of $K B$.

$(\Rightarrow)$ Let $I_{\nu}$ be a $\nu$-model of $K B$. We define $\mathcal{E}=\left\{\left(n_{i}, n_{j}\right) \mid n_{i}{ }^{I_{\nu}}=n_{j}{ }^{I_{\nu}}\right\}$ and construct a $\pi$-interpretation $I_{\pi}$ by setting $\triangle^{I_{\pi}}=\triangle^{I_{\nu}},(n / \mathcal{E})^{I_{\pi}}=n^{I_{\nu}}$, $C^{I_{\pi}}(n / \mathcal{E})=C^{I_{\nu}}\left(n^{I_{\nu}}\right)$ and $R^{I_{\pi}}(n / \mathcal{E})=R^{I_{\nu}}\left(n^{I_{\nu}}\right)$, for each $n \in N_{K B}$. Again, for each concept $X, C^{I_{\pi}}(X / \mathcal{E})=C^{I_{\nu}}(X)$, so $I_{\pi}$ is a $\pi$-model of $K B / \mathcal{E}$.

Now Lemma 2 immediately implies the following result:

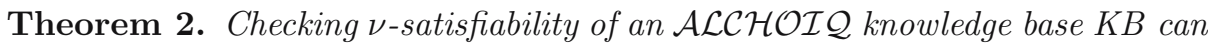
be performed in non-deterministic exponential time, assuming numbers are coded in unary.

Proof. Observe that $\left|N_{K B}\right|$ is linear in $|K B|$, and that each equivalence relation $\mathcal{E}$ is a subset of $N_{K B} \times N_{K B}$. Hence, the number of possible equivalence relations is exponential in $|K B|$. A decision procedure for checking $\nu$-satisfiability of $K B$ can systematically examine all equivalence relations $\mathcal{E}$ and for each one perform a $\pi$ satisfiability check of $K B / \mathcal{E}$. The last step can be performed in non-deterministic exponential time, since $\mathcal{A L C H O I} \mathcal{Q}$ is a fragment of $\mathcal{C}^{2}$ - the two-variable firstorder logic with counting, which is decidable in NExPTIME assuming numbers are coded in unary [12. Hence, the overall algorithm runs in non-deterministic exponential time as well.

We briefly compare the results of Theorems 1 and 2. The main feature of $\nu$-semantics is the reification of concept and role names. However, it is more like $\pi$-semantics and less like OWL-Full semantics in the way it handles the modeling primitives. In particular, in $\nu$ - and $\pi$-semantics, these are expressed as formulae and are not accessible as individuals in the knowledge base. On the contrary, OWL-Full reifies the modeling primitives as well, and thus allows their semantics to be altered by the statements in the knowledge base.

\subsection{HiLog Semantics and Transitivity}

The differences between the algorithms for checking $\nu$ - and $\pi$-satisfiability are minor. Since the latter algorithm can easily handle transitive roles, one might expect the former one to be easily extended to handle transitivity as well. However, consider the following knowledge base $K B$ : 


$$
\begin{array}{r}
\top \sqsubseteq \geq 3 S \\
S \approx T \\
\operatorname{Trans}(T)
\end{array}
$$

Notice that $K B$ is a $\mathcal{S H O I} \mathcal{Q}$ knowledge base: the role $S$ is simple, since it passes the syntactic criterion specified in Definition 2 (i.e., it is neither transitive nor it has transitive subroles). However, in any $\nu$-interpretation $I$, (13) ensures that $S^{I}=T^{I}=\alpha$. Furthermore, due to (14), $R^{I}(\alpha)$ is transitive. Effectively, in (12) a transitive role is used in a number restriction, even though $S$ is syntactically a simple role.

Since equality of role names might be non-trivially entailed by $K B$, identifying this requires theorem proving itself. This makes a check for simple roles under $\nu$-semantics difficult, if not impossible. Because allowing transitive roles in number restrictions leads to undecidability [7], we get the following result:

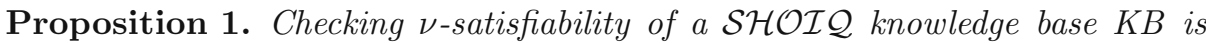
undecidable.

Decidability can be regained by using unique role assumption, requiring symbols used as roles in $K B$ to be interpreted as distinct domain individuals.

Definition 5 (Unique Role Assumption). A SHOI $\mathcal{S}$ knowledge base $K B$ employs unique role assumption (URA) if it contains an axiom $S \not T$ for each two distinct names $S$ and $T$ occurring as roles in $K B$.

If $K B$ employs URA or if it contains neither explicit equality statements nor number restrictions, role interpretations of different symbols can be assumed to be independent. Then, simple roles can be checked as usual, and transitivity axioms of $K B$ can be eliminated by transforming $K B$ into an equisatisfiable $\mathcal{A L C H O I Q}$ knowledge base $\Omega(K B)$, as done in [10]. Roughly speaking, a transitivity axiom $\operatorname{Trans}(S)$ is replaced with axioms of the form $\forall R . C \sqsubseteq \forall S .(\forall S . C)$, for each $R$ with $S \sqsubseteq^{*} R$ and $C$ a concept occurring in $K B$. This transformation is polynomial, so it does not increase the complexity of reasoning. Hence, $\nu$-satisfiability of a $\mathcal{S H O I} \mathcal{Q}$ knowledge base $K B$ employing URA can be deiced by checking $\nu$-satisfiability of the $\mathcal{A L C H O I} \mathcal{Q}$ knowledge base $\Omega(K B)$.

\subsection{A Practical Reasoning Procedure for $\mathcal{A} \mathcal{L C H} \mathcal{H} \mathcal{Q}$}

The reasoning procedure from Section 3.2 is worst-case optimal, but is unlikely to be effective in practice, since it systematically examines exponentially many equivalence relations. Therefore, we now present a practical, resolution-based

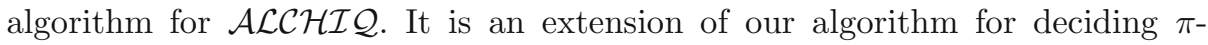
satisfiability from [10], and extending it to handle nomials is part of our ongoing work. Using the transformation of transitivity axioms from the previous subsection, this algorithm can also decide $\nu$-satisfiability of $\mathcal{S H \mathcal { I }}$ knowledge bases. Due to space constraints, we omit many technical details, which are given in $[9]$. We assume familiarity with first-order logic and resolution theorem proving. 
Table 3. $\nu$-semantics by Mapping into First-order Logic

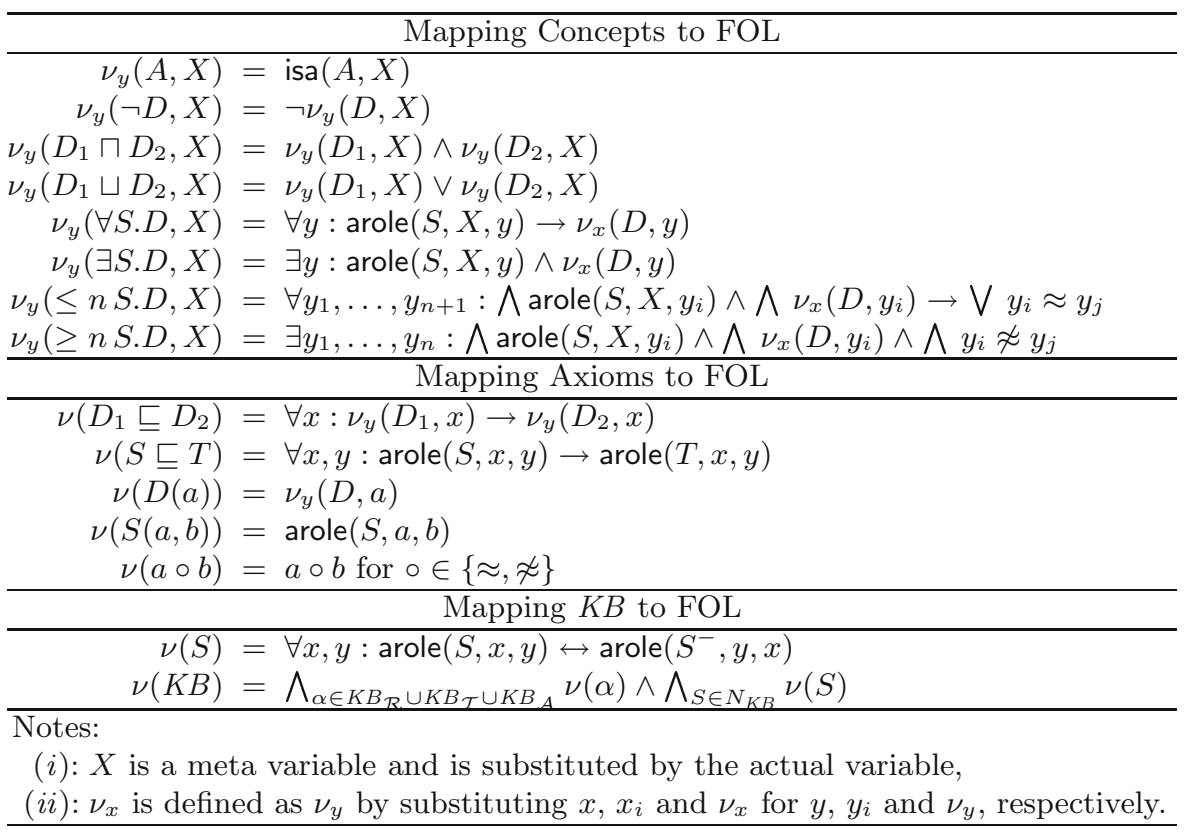

Translation into First-order Logic. Our algorithm is based on resolution, so in Table 3 we define an operator $\nu$ which translates $K B$ into a formula $\nu(K B)$ of first-order logic with equality. As shown by the following lemma, $\nu(K B)$ is first-order satisfiable if and only if $K B$ is $\nu$-satisfiable. Intuitively, for a name $n$, isa $(n, x)$ encodes the concept extension of $n$ and arole $(n, x, y)$ encodes the role extension of $n$. Therefore, a $\nu$-interpretation $I_{\nu}$ of $K B$ can be easily converted into a first-order interpretation $I$ of $\nu(K B)$ and vice versa.

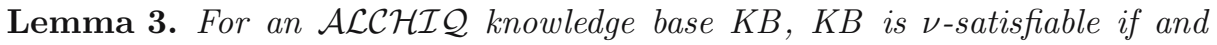
only if a first-order model of $\nu(K B)$ exists.

Basic Superposition Calculus. We decide first-order satisfiability of $\nu(K B)$ by basic superposition [2] $(\mathcal{B S})$, a clausal calculus optimized for theorem proving with equality. The calculus is parameterized by a certain term ordering and a selection function. It consists of resolution and superposition rules, which are applied only to literals in clauses designated by the chosen parameters. A set of clauses $N$ is saturated by $\mathcal{B S}$ if applying a rule of $\mathcal{B S}$ to premises from $N$ produces an already derived clause. $\mathcal{B S}$ is sound and complete: a saturated set of clauses $N$ is unsatisfiable if and only if it contains the empty clause.

Decision Procedure by $\mathcal{B S}$. In order to apply $\mathcal{B S}$, we transform $\nu(K B)$ into the set of clauses $\Xi_{\nu}(K B)$ using the so-called structural transformation [1], which ensures that this step is polynomial. 
We now saturate $\Xi_{\nu}(K B)$ by $\mathcal{B S}_{D L}$, where $\mathcal{B S}_{D L}$ denotes the $\mathcal{B S}$ calculus parameterized as discussed in 9. It is possible to show that during such a saturation, only clauses of a certain syntactic form are derivable, and that the number of possible derived clauses is exponential in $|K B|$. Therefore, saturation by $\mathcal{B S}_{D L}$ will terminate after an exponential number of steps. Since $\mathcal{B S}_{D L}$ is sound and complete, it decides satisfiability of $\Xi_{\nu}(K B)$ and, by Lemma 3 , $\nu$ satisfiability of $K B$. The actual algorithm has to deal with several techical issues, for which we direct the reader to 9 . Hece we just state our main result:

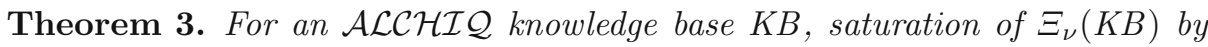
$\mathcal{B S}_{D L}$ decides $\nu$-satisfiability of $K B$ and runs in time exponential in $|K B|$, assuming numbers are coded in unary.

\section{Expressivity of Metamodeling}

We now discuss the benefits of metamodeling in terms of additional consequences that can be drawn. These results are similar to the ones for HiLog from [4.

It is easy to see that $\nu$-satisfiability is a strictly stronger notion than $\pi$ satisfiability. Consider the following knowledge base $K B$ :

$$
\begin{array}{r}
\text { Eagle }(\text { Harry }) \\
\neg \text { Aquila }(\text { Harry }) \\
\text { Eagle } \approx \text { Aquila }
\end{array}
$$

Under the contextual semantics, the interpretations of the symbols Eagle and Aquila as concepts and as individuals are independent, so $K B$ is $\pi$-satisfiable. However, $K B$ is $\nu$-unsatisfiable: in each $\nu$-interpretation Eagle $^{I}=$ Aquila $^{I}=\alpha$, so it cannot be that Harry $^{I} \in C^{I}\left(\right.$ Eagle $\left.^{I}\right)$ and Harry ${ }^{I} \notin C^{I}\left(\right.$ Aquila $\left.^{I}\right)$. For the other direction, we have the following lemma:

Lemma 4. A $\nu$-satisfiable $\mathcal{S H O I Q ~ k n o w l e d g e ~ b a s e ~} K B$ is also $\pi$-satisfiable.

Proof. Let $I_{\nu}$ be a $\nu$-model of an $\mathcal{S H O I Q}$ knowledge base $K B$. We construct a $\pi$-interpretation $I_{\pi}$ as follows: $\triangle^{I_{\pi}}=\triangle^{I_{\nu}}, n^{I_{\pi}}=n^{I_{\nu}}, C^{I_{\pi}}(n)=C^{I_{\nu}}\left(n^{I_{\nu}}\right)$ and $R^{I_{\pi}}(n)=R^{I_{\nu}}\left(n^{I_{\nu}}\right)$, for each $n \in N_{K B}$. By a straightforward induction on the concept structure it can be shown that, for each concept $X, C^{I_{\pi}}(X)=C^{I_{\nu}}(X)$, so $I_{\pi}$ is a $\pi$-model of $K B$.

Furthermore, for a knowledge base with unique name assumption or without equality (either explicit or implicit, introduced through number restrictions), $\pi$-satisfiability and $\nu$-satisfiability coincide:

Lemma 5. Let $K B$ be an $\mathcal{S H O I Q ~ k n o w l e d g e ~ b a s e ~ s u c h ~ t h a t ~ i t ~ e m p l o y s ~ u n i q u e ~}$ name assumption, or it contains neither explicit equality statements nor number restrictions. Then $K B$ is $\pi$-satisfiable if and only if it is $\nu$-satisfiable.

2 "Aquila" is the Latin name for "eagle." 
Proof. The $(\Leftarrow)$ direction follows from Lemma 4 For the $(\Rightarrow)$ direction, let $K B$ be $\pi$-satisfiable in some model $I_{\pi}$. Since $K B$ either employs unique name assumption or it does not employ equality, without loss of generality, we may assume that for each $n_{i}, n_{j} \in N, n_{i} \neq n_{j}$ implies $n_{i}{ }^{I_{\pi}} \neq n_{j}{ }^{I_{\pi}}$.

We now construct a $\nu$-interpretation $I_{\nu}$ as follows: $\triangle^{I_{\nu}}=\triangle^{I_{\pi}}, n^{I_{\nu}}=n^{I_{\pi}}$, $C^{I_{\nu}}\left(n^{I_{\nu}}\right)=C^{I_{\pi}}(n)$ and $R^{I_{\nu}}\left(n^{I_{\nu}}\right)=R^{I_{\pi}}(n)$, for $n \in N_{K B}$. Furthermore, for all $x \in \triangle^{I_{\nu}}$ such that there is no $n \in N_{K B}$ with $x=n^{I_{\nu}}$, let $C^{I_{\nu}}(x)=R^{I_{\nu}}(x)=\emptyset$. Since we can assume that different names of $N_{K B}$ are interpreted as different elements of $\triangle^{I_{\nu}}$, the construction assigns a unique value to $C^{I_{\nu}}(x)$ and $R^{I_{\nu}}(x)$ for each $x \in \triangle^{I_{\nu}}$, so $I_{\nu}$ is correctly defined. By a straightforward induction on the concept structure it can be shown that, for each concept $X, C^{I_{\nu}}(X)=C^{I_{\pi}}(X)$. Therefore, $I_{\nu}$ is a $\nu$-model of $K B$.

To summarize, $\nu$-semantics allows deriving new consequences only if it is possible to derive that two symbols are equal; for example, from (15) and (17) it is possible to derive Aquila(Harry). Furthermore, if the unique name assumption is employed, as it is often the case in practice, $\nu$-semantics does not yield any additional consequences. This seems to suggest that the benefits of $\nu$-semantics do not outweigh its drawbacks, namely, the fact that it is non-standard and that it introduces problems for transitive roles. Moreover, $\pi$-semantics might be sufficient for many practical applications.

However, $\nu$-semantics unlocks its full potential when combined with a language more expressive than OWL. For example, by combining $\nu$-semantics with the Semantic Web Rule Language (SWRL) [6], one can explicitly axiomatize the semantics of metaconcepts. Consider the example from Section 1 By (18) we state that Eagle is an RedListSpecies, and by a SWRL rule (19) we state that instances of species listed in the Red List are not allowed to be hunted. Notice that in atom $S(I)$ we use the variable $S$ at the position of a predicate. Under $\nu$-semantics this is equivalent to isa $(S, I)$, but under $\pi$-semantics this would not be possible without leaving the confines of first-order logic. Now from (15), (18) and (19), we may infer CannotHunt(Harry), so RedListSpecies semantically acts as a metaconcept of the Eagle concept.

$$
\begin{array}{r}
\text { RedListSpecies }(\text { Eagle }) \\
\operatorname{RedListSpecies~}(S) \wedge S(I) \rightarrow \text { CannotHunt }(I)
\end{array}
$$

To summarize, from the logical perspective, $\nu$-semantics alone does not bring much, and $\pi$-semantics may be sufficient for numerous applications. However, $\nu$-semantics provides a sound foundation for metamodeling, which, when combined with expressive logical formalisms such as SWRL, allows precisely axiomatizing the interaction between concepts and metaconcepts. Thus, we believe $\nu$-semantics to be very relevant for the future extensions of OWL.

\section{Related Work}

The definition of $\nu$-satisfiability given in Section 3 is inspired by HiLog 4, a logic in which general terms are allowed to occur in place of function and predicate 
symbols in formulae. The semantics is defined by interpreting each individual as a member of the interpretation domain, and by assigning a functional and a relational interpretation to domain objects. The authors show that HiLog can be considered "syntactic sugar", since each HiLog formula can be encoded into an equisatisfiable first-order formula. The definition of the $\nu$ operator in Table 2 closely resembles this encoding. Finally, the authors show that a satisfiable firstorder formula without equality is also satisfiable under HiLog semantics.

In [13, the RDFS Model Theory was criticized for allowing infinite number of meta-layers. The authors argue that such semantics is inadequate for the Semantic Web because $(i)$ it does not provide adequate support for inferencing, $(i i)$ it allows defining classes containing themselves, which may lead to paradoxes, and ( iii) by adding classes, one necessarily introduces objects in the interpretation universe. The authors propose RDFS-FA, a stratified four-level approach, consisting of the meta-language layer, the language layer, the ontology layer and the instance layer. In [5] similar arguments were used to criticize the semantics of OWL-Full. We follow the principles of RDFS-FA by strictly separating the modeling primitives from the ontology and the instance layers. However, to allow metamodeling, our definition of $\nu$-semantics merges the ontology and the instance layers into one. Furthermore, we show that (iii) affects the logical consequences only if equality reasoning is required, which matches well with the intuition behind metamodeling.

In [16] the authors point out the usefulness of metamodeling in many application domains. They propose separation of modeling layers, which are connected using so-called spanning instances. However, the authors do not consider the logical consequences of their approach.

\section{Conclusion}

In this paper we have analyzed the metamodeling features of OWL-Full, the most expressive of the Semantic Web ontology languages. We have shown that the style of metamodeling adopted in OWL-Full leads to undecidability of basic reasoning problems, due to mixing logical and metalogical primitives. In order to obtain a decidable and expressive language supporting metamodeling, we have proposed two alternative semantics: the contextual one, which is essentially first-order, and the HiLog one, which is more in the spirit of OWL-Full. Under certain technical assumptions, both semantics are decidable when combined with the description logic $\mathcal{S H O I} \mathcal{H}$. Furthermore, we have presented a

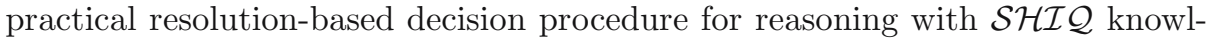
edge bases under HiLog semantics, thus obtaining practical support for a logic with metamodeling whose expressivity is between OWL-Lite and OWL-DL.

We have analyzed the added expressivity of metamodeling and have shown that the HiLog semantics allows deriving new conclusions only by equality reasoning. However, this approach unlocks its full potential if combined with expressive extensions, such as SWRL, since it allows axiomatizing the logical interaction between concepts and their metaconcepts. 
In future, we shall attempt to extending the practical decision procedure from Subsection 3.4 to handle nominals as well, and thus to cover all of OWL-DL.

\section{Acknowledgements}

This work was partially funded by the EU IST project DIP 507483. We thank the anonymous reviewer for valuable comments regarding Subsection 3.2

\section{References}

1. F. Baader, D. Calvanese, D. McGuinness, D. Nardi, and P. Patel-Schneider, editors. The Description Logic Handbook. Cambridge University Press, January 2003.

2. L. Bachmair, H. Ganzinger, C. Lynch, and W. Snyder. Basic Paramodulation. Information and Computation, 121(2):172-192, 1995.

3. R. Berger. The undecidability of the dominoe problem. Memoirs of the American Mathematical Society, 66, 1966.

4. W. Chen, M. Kifer, and D. S. Warren. HILOG: a foundation for higher-order logic programming. Journal of Logic Programming, 15(3):187-230, 1993.

5. I. Horrocks and P. F. Patel-Schneider. Three Theses of Representation in the Semantic Web. In Proc. WWW 2003, pages 39-47. ACM, 2003.

6. I. Horrocks and P. F. Patel-Schneider. A Proposal for an OWL Rules Language. In Proc. $W W W$ 2004. ACM, 2004.

7. I. Horrocks, U. Sattler, and S. Tobies. Practical Reasoning for Very Expressive Description Logics. Logic Journal of the IGPL, 8(3):239-263, 2000.

8. I. Horrocks, U. Sattler, and S. Tobies. Reasoning with Individuals for the Descrip-

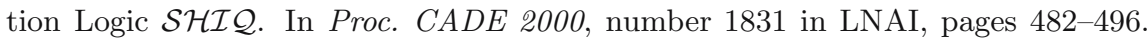
Springer, 2000.

9. U. Hustadt, B. Motik, and U. Sattler. Reasoning for Description Logics around

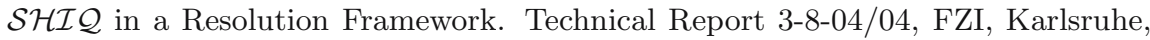
Germany, April 2004. http://www.fzi.de/ipe/publikationen.php?id=1172.

10. U. Hustadt, B. Motik, and U. Sattler. Reducing $\mathcal{S H} \mathcal{H} \mathcal{Q}^{-}$Description Logic to Disjunctive Datalog Programs. In Proc. KR 2004, pages 152-162, Menlo Park, California, USA, June 2004. AAAI Press.

11. A. Nonnengart and C. Weidenbach. Computing Small Clause Normal Forms. In A. Robinson and A. Voronkov, editors, Handbook of Automated Reasoning, volume I, chapter 6, pages 335-367. Elsevier Science, 2001.

12. L. Pacholski, W. Szwast, and L. Tendera. Complexity Results for First-Order Two-Variable Logic with Counting. SIAM Journal on Computing, 29(4):10831117, 2000.

13. J. Pan and I. Horrocks. RDFS(FA) and RDF MT: Two Semantics for RDFS. In Proc. ISWC 2003, number 2870 in LNCS, pages 30-46. Springer, 2003.

14. P. F. Patel-Schneider, P. Hayes, and I. Horrocks. OWL Web Ontology Language; Semantics and Abstract Syntax. http://www.w3.org/TR/owl-semantics/, 2002.

15. G. Schreiber. The Web is not well-formed. IEEE Intelligent Systems, 17(2):79-80, March/April 2002. Contribution to the section "Trends \& Controversies: Ontologies KISSES in Standardization", edited by S. Staab.

16. C. Welty and D. Ferrucci. What's in an instance? Technical Report 94-18, MaxPlanck-Institut, 1994. RPI Computer Science. 